\title{
Toxicidad ocular medicamentosa
}

\section{Ocular toxicity induced by medication}

\author{
A. Garralda
}

\section{RESUMEN}

En el presente trabajo se analiza la toxicidad ocular inducida por cuatro grupos de fármacos sistémicos: tamoxifeno, cloroquina y derivados, anticolinérgicos y tamsulosina. El objetivo principal es dar unas normas prácticas que sirvan para el manejo diario de los pacientes sometidos a estos tratamientos, más que profundizar en los mecanismos de acción de cada uno de ellos.

Palabras clave. Tamoxifeno. Hidroxicloroquina. Anticolinérgicos. Tamsulosina. Ifis.

An. Sist. Sanit. Navar. 2008; 31 (Supl. 3): 147-153.

\begin{abstract}
This paper analyses ocular toxicity induced by four groups of systemic medicines: tamoxifen, chloroquine and derivatives, anticholinergics and tamsulosin. Our main aim is to provide some practical rules that will be of use in the everyday management of patients receiving these treatments, rather than to examine the mechanisms by which each of these medicines act.
\end{abstract}

Palabras clave. Tamoxifen. Hydroxichloroquine. Anticholinergics. Tamsulosin. Ifis.
Servicio de Oftalmología. Hospital Virgen del Camino. Pamplona.

\section{Correspondencia:}

Andrés Garralda Luquin

Servicio de Oftalmología

Hospital Virgen del Camino

Irunlarrea, 4

31008 Pamplona

Tfno. 848422606

E-mail: andres.garralda.luquin@cfnavarra.es 


\section{INTRODUCCIÓN}

Se han descrito complicaciones oculares de casi todos los fármacos existentes. La mayoría son anecdóticas, pero unas pocas pueden tener gran importancia por su frecuencia o gravedad. No es el objetivo de este trabajo detallarlas todas. Sería, además de tedioso, poco práctico por varios motivos: el medicamento ya no se usa o se usa muy poco, no se ha probado claramente la relación causal, la dosis necesaria para que se produzca el efecto secundario es tan alta que nunca se alcanza en la vida real, etc.

Sólo serán tratados en profundidad cuatro fármacos. Los criterios que se han elegido para seleccionarlos han sido los siguientes: que se usen con mucha frecuencia (tamoxifeno), que puedan provocar complicaciones quirúrgicas (alfabloqueantes y síndrome del iris flácido intraoperatorio), que no esté claro si pueden administrarse en pacientes con patología ocular (fármacos con efecto anticolinérgico y glaucoma) y que sus efectos oculares sean graves pero evitables (cloroquina y derivados). Como complemento, y para que puedan realizarse consultas rápidas, se ha añadido una tabla (Tabla 1) que incluye algunos medicamentos y sus efectos adversos.

Por último, señalar que en el artículo se emplean, en ocasiones, descripciones muy simples de procesos complejos. Se ha hecho así para que resulte comprensible al profesional médico no oftalmológico.

\section{TAMOXIFENO}

El tamoxifeno es un antagonista estrogénico no esteroideo empleado como tratamiento coadyuvante en los adenocarcinomas de mama con receptor estrogénico positivo. Se administra por vía oral, en muchos casos durante cinco o más años.

Desde 1974 se sabe que puede provocar retinopatía ${ }^{1}$. Otros efectos adversos oculares son los depósitos corneales, la neuritis óptica y la catarata. Cuando se describió la retinopatía, las pacientes eran tratadas con dosis muy altas de tamoxifeno (del orden de $180 \mathrm{mg} /$ día). Los hallazgos que se encontraron fueron: pequeños depósitos brillantes blanquecinos en la retina interna, especialmente en el área paramacular, alteraciones del epitelio pigmentario y edema de mácula. Las dosis usadas hoy en día son mucho más bajas y es raro encontrar formas tan severas. Aunque la incidencia de retinopatía es variable según los estudios ${ }^{24}$, se puede estimar en un 5\% después de un año de terapia. Las manifestaciones clínicas tampoco son siempre iguales. Hay casos en los que pese a detectarse oftalmoscópicamente depósito macular el paciente está asintomático. Otras veces, en cambio, aparece dificultad en el reconocimiento de los colores e incluso pérdida de agudeza visual.

Se desconoce el mecanismo exacto por el que el tamoxifeno induce toxicidad ocular. Es posible que, al poseer uniones polares y apolares con lípidos que no son metabolizados, se acumule en los complejos intracelulares de los lisosomas.

No está claro si la disminución de visión que produce la retinopatía por tamoxifeno es reversible ${ }^{5}$ o no ${ }^{6}$. Tampoco se ha establecido un consenso sobre si, en el caso de que aparezcan trastornos oculares, debe interrumpirse la medicación (por la amenaza para la visión) o no (por el riesgo de reducir la supervivencia) ${ }^{7}$. No obstante, a continuación damos unas pautas recogidas de la bibliografía ${ }^{8,9}$ que pueden resultar útiles en la práctica. Así se ha de:

- Hacer un estudio basal oftalmológico antes de iniciar el tratamiento.

- Hacer controles anuales oculares si no surgen complicaciones.

- Incrementar la frecuencia de las revisiones oftalmológicas (trimestrales) en caso de que aparezcan cristales en la retina pero sin otros signos o síntomas. No es necesario interrumpir la terapia.

- Suspender el tratamiento si se detecta disminución de la visión.

\section{CLOROQUINA E HIDROXICLOROQUINA}

La primera indicación de la cloroquina fue el tratamiento y la profilaxis de la malaria. Más tarde se le encontraron otros usos de interés clínico. Los reumatólogos la utilizan con éxito en la artritis reumatoide y 
Tabla 1. Reacción de fármacos y reacciones adversas más frecuentes.

\begin{tabular}{|c|c|}
\hline Fármaco & Reacción adversa \\
\hline Ácido nalidíxico & Alteración de la visión cromática. Pseudotumor cerebral \\
\hline Ácido nicotínico & Edema macular \\
\hline Adriamicina & Pigmentación rojiza de la lágrima \\
\hline Alcohol metílico & Atrofia óptica \\
\hline Alopurinol & Cataratas. Hemorragias maculares \\
\hline Amantadina & Alucinaciones visuales \\
\hline Amiodarona & Córnea verticillata \\
\hline Anticoagulantes & Hemorragias intraoculares \\
\hline Anticonceptivos orales & Hemorragias retinianas. Cataratas \\
\hline Bupivacaína & Hemorragias retinianas \\
\hline Busulfán & Catarata subcapsular posterior \\
\hline Carbamacepina & Pigmentación retiniana. Catarata \\
\hline Carboplatino & Ceguera cortical \\
\hline Ciprofloxacino & Visión borrosa. Neuropatía óptica \\
\hline Cisplatino & Ceguera cortical \\
\hline Cloracepato dipotásico & Nistagmo \\
\hline Clorambucil & Queratitis. Hemorragias retinianas. Diplopia \\
\hline Cloramfenicol & Neuropatía óptica. Retinopatía \\
\hline Clorpropamida & Midriasis. Errores de refracción. Diplopia. Neuropatía \\
\hline Corticoides & Catarata. Coriorretinopatía serosa central. Glaucoma \\
\hline Estreptomicina & Neuropatía óptica \\
\hline Etambutol & Neuropatía óptica \\
\hline Ibuprofeno & Neuropatía óptica \\
\hline Interferón & Retinopatía. Hemorragia subconjuntival \\
\hline Isoniacida & Neuropatía óptica \\
\hline Metoclopramida & Nistagmo. Diplopia \\
\hline Metotrexato & Fotofobia. Hiperemia conjuntival \\
\hline Naproxeno & Opacidades corneales. Neuropatía óptica \\
\hline Oxígeno & Retinopatía del prematuro \\
\hline Practolol & Queratopatía \\
\hline Rifabutina & Uveitis anterior \\
\hline Sales de oro & Depósitos corneales \\
\hline Salicilatos & Midriasis. Hemorragias retinianas. Nistagmo. Neuropatía \\
\hline Tetraciclinas & Pseudotumor cerebral \\
\hline Trimetadiona & Diplopia. Nistagmo. Conjuntivitis \\
\hline Vincristina & Neuropatía óptica. Diplopia \\
\hline Vitamina A & Pseudotumor cerebral \\
\hline Vitamina D & Opacificaciones corneales. Estrabismo \\
\hline
\end{tabular}

Datos publicados por Fontenla y $\mathrm{col}^{20}$.

otras enfermedades del tejido conectivo y los dermatólogos la emplean en el lupus cutáneo. La hidroxicloroquina comparte el mismo perfil terapéutico que la cloroquina, pero es la que más se utiliza en la actualidad por poseer menor toxicidad.

Aunque es posible encontrar depósitos de hidroxicloroquina en la córnea, el principal efecto secundario ocular es la retinopatía. Ésta se ha descrito, en casos muy evolucionados, como maculopatía en ojo de buey por el aspecto oftalmoscópico que ofrece. La pérdida de la visión, si no se interrumpe el tratamiento, llega a ser grave e irreversible. No obstante, comparada con otros fármacos empleados en las enfermedades reumáticas, se considera un tratamiento bien tolerado y de fácil monitorización. Además, a las dosis empleadas en la actualidad (menores a $6 \mathrm{mg} / \mathrm{kg} /$ día) la retinopatía es muy poco frecuente. El riesgo de desarrollarla aumenta si la dosis acumulada supera los 800 gramos y si el paciente tiene más de 70 años $^{10}$. El mecanismo exacto por el que se produce no es bien conocido, aunque pueden estar impli- 
cados varios factores, como el estrés oxidativo y las mutaciones del gen $\mathrm{ABCR}^{11}$.

Para la práctica diaria, el aspecto más relevante a tener en cuenta es determinar qué pruebas de control, y con qué frecuencia deben hacerse para diagnosticar precozmente la maculopatía. A pesar de los muchos años que se lleva usando la hidroxicloroquina, todavía no se ha llegado a un acuerdo completo. Posiblemente, la guía dada por el Royal Collage of Ophthalmologist del Reino Unido es la más aceptada por la comunidad médica. De forma resumida recomienda:

- Antes de iniciar tratamiento se debe tener un conocimiento claro del estado ocular del paciente, empleando para ello los medios que se consideren oportunos (historia clínica, optometrista y oftalmólogo).

- Una vez que el paciente esté tomando el fármaco habrá que revisarle la agudeza visual e interrogarle sobre síntomas visuales una vez al año. Esto puede hacerlo un médico de Atención Primaria o un óptico.

- Si en algún momento se objetiva disminución de agudeza visual o si el paciente refiere síntomas visuales deberá interrumpirse la toma de la medicación hasta que el enfermo sea sometido a valoración por un oftalmólogo.

- Cuando el tratamiento vaya a durar más de 5 años se establecerá, individualmente para cada paciente, una pauta de controles oftalmológicos.

- La exploración oftalmológica de estos pacientes debe incluir: agudeza visual próxima y lejana, visión cromática, campo visual, rejilla de Amsler, biomicroscopía y funduscopia.

Aunque las normas dadas arriba son las más aceptadas, siguen sin serlo de forma unánime. Además, éstas se completan con la realización de una campimetría a los dos años de iniciado el tratamiento y anualmente después ${ }^{12}$.

\section{FÁRMACOS CON EFECTO ANTICOLINÉRGICO Y GLAUCOMA}

Muchos fármacos de uso sistémico presentan acción anticolinérgica. En ocasio- nes se busca este efecto (por ejemplo, el bromuro de ipratropium empleado en bronquíticos), pero en la mayoría de los casos es una fuente de reacciones adversas no deseadas (por ejemplo, en los antihistamínicos, en los antiparkinsonianos, en los antiarrítmicos, etc.). Una de estas posibles reacciones adversas es la aparición de un glaucoma agudo de ángulo cerrado.

Antes de analizar las posibles consecuencias de estos medicamentos en los ojos se van a repasar de forma muy breve los dos tipos principales de glaucoma: el de ángulo abierto y el de ángulo cerrado.

Para el adecuado funcionamiento del ojo es necesario que se renueve el humor acuoso. Este líquido se produce en los procesos ciliares, llena la cámara posterior (espacio comprendido entre el cristalino y el iris), pasa por la pupila a la cámara anterior (espacio entre el iris y la córnea) y sale del ojo, principalmente, a través de la malla trabecular situada en el ángulo iridocorneal. Ésta puede obstruirse poco a poco porque se acumulen depósitos (lo que denominamos aumento de la resistencia a la salida del acuoso), o puede tener un diámetro tan pequeño que se cierre del todo si aparece alguna circunstancia que reduzca aún más ese diámetro. En el primer caso tendríamos un glaucoma crónico de ángulo abierto (la tensión ocular sube lentamente en un espacio de tiempo prolongado. Aunque el paciente está, aparentemente, asintomático su nervio óptico está sufriendo un daño continuo) y en el segundo, un glaucoma agudo de ángulo cerrado (la tensión sube bruscamente, el enfermo tiene dolor intenso, náuseas e incluso puede perder la visión de forma irreversible en unas horas).

Las personas que desarrollan un glaucoma agudo tienen una predisposición anatómica para ello: un ángulo camerular estrecho. El factor que desencadena el glaucoma es, la mayoría de las veces, la midriasis pupilar.

La miosis pupilar es dependiente de la inervación parasimpática y la midriasis de la simpática. Por lo tanto, si a una persona le administramos un anticolinérgico (aunque sea por vía sistémica) se le va a indu- 
cir una midriasis. Si ese individuo tiene un ángulo muy estrecho puede que se le provoque un glaucoma agudo.

Es importante señalar que, aunque se han publicado algunos artículos que intentan relacionar los fármacos anticolinérgicos sistémicos con la elevación de la tensión en ojos con ángulo abierto ${ }^{13}$, no hay pruebas de que los anticolinérgicos produzcan empeoramiento de los pacientes con glaucoma crónico simple. Por lo tanto, aquellos enfermos que estén siendo tratados de este tipo de glaucoma (con betabloqueantes, prostaglandinas o cualquier otro antiglaucomatoso) y requieran tratamiento con fármacos sistémicos de acción anticolinérgica (por ejemplo, un antidepresivo) pueden iniciarlo sin temor a que ocurra nada malo.

Lo verdaderamente peligroso es emplear un fármaco con efecto anticolinérgico en un paciente con ángulo estrecho. En este caso, la relación causal entre la administración del medicamento y la aparición de glaucoma agudo es clara y se ha descrito en muchas ocasiones ${ }^{14,15}$.

A continuación, y teniendo en cuenta lo explicado anteriormente, se dan unas pautas prácticas para aquellos médicos no oftalmólogos que tengan que prescribir fármacos sistémicos con efecto anticolinérgico:

- Se pueden emplear sin temor en pacientes que han sido vistos por un oftalmólogo y catalogados como normales.

- Pueden utilizarse con normalidad en enfermos diagnosticados de glaucoma crónico de ángulo abierto. No obstante, es aconsejable que se informe al oftalmólogo de que se han prescrito.

- Pueden utilizarse en pacientes con diagnóstico de ángulo estrecho a los que se les han aplicado las medidas profilácticas para evitar el glaucoma agudo (por ejemplo, la iridotomía YAG).

- Si desconocemos el estado ocular del paciente podemos hacer una estimación aproximada de la profundidad de la cámara anterior (lo que guarda paralelismo con la abertura del ángulo iri- docorneal) valiéndonos de una linterna. Basta con situar esta linterna junto al limboesclerocorneal temporal y dirigir la luz paralela al plano del iris. Si la cámara anterior es profunda la superficie del iris se iluminará en su totalidad y de forma uniforme. En este caso podemos administrar el anticolinérgico. Si tenemos dudas o vemos claramente que la cámara es estrecha lo mejor es no pautar el medicamento y remitir al paciente a un especialista para que le haga una exploración más completa.

\section{SÍNDROME DEL IRIS FLÁCIDO INTRAOPERATORIO}

Más conocido como IFIS (Intraoperative Floppy Iris Syndrome), es una triada quirúrgica que incluye ondulaciones del estroma del iris, propensión al prolapso de iris y constricción pupilar. Inicialmente se describió asociada al uso de la tamsulosina ${ }^{16}$, un fármaco empleado en el tratamiento de la hipertrofia benigna de próstata. Aunque puede presentarse con otros antagonistas de los receptores $\beta 1$ adrenérgicos, suele hacerlo con menos frecuencia e intensidad.

La importancia de este síndrome radica en que se asocia a un incremento de las complicaciones en la cirugía de la catarata. Fuera del acto quirúrgico, el efecto de estos fármacos sobre el iris carece de trascendencia clínica.

En la actualidad y en nuestro medio, las cataratas se operan utilizando la técnica de la facoemulsificación. Esta técnica consiste, básicamente, en introducir en el ojo un instrumento (vulgarmente llamado "Faco") que destruye y aspira el cristalino. La punta del Faco entra en el globo ocular a través de una incisión en la córnea y alcanza el cristalino después de pasar por la pupila. Cuanto más dilatada esta la pupila mejor se ve la catarata y más sencillas resultan las maniobras con el Faco. A mayor miosis, mayor riesgo de complicaciones.

Si administramos un fármaco que bloquea los receptores $\beta 1$ adrenérgicos (tamsulosina) vamos a dificultar la midriasis y, por consiguiente, vamos a incrementar el 
riesgo de que surjan problemas quirúrgicos.Visto lo anterior, podría pensarse que lo mejor es no prescribir estos fármacos o suspenderlos si se están usando. Sin embargo, esto no es así. En primer lugar, la relación beneficio/riesgo es favorable al empleo del medicamento. En segundo, se ha descrito IFIS tres años después de retirar la tamsulosina. La actitud más adecuada será una actuación individualizada. No obstante, damos a continuación algunos consejos extraídos de la bibliografía ${ }^{17-19}$ :

- Explicar al enfermo los riesgos quirúrgicos asociados al empleo de estos medicamentos.

- En pacientes que no han recibido tratamiento para la hipertrofia benigna de próstata y tienen una catarata operable, hacer primero la cirugía e iniciar el tratamiento con tamsulosina, o similares, después.

- Una vez iniciado el tratamiento no merece la pena discontinuarlo unas semanas antes de la cirugía: normalmente no se consigue nada.

- En el momento de la operación pueden ser útiles algunas de estas maniobras: dilatación prequirúrgica con colirio de atropina; si se sospecha que el iris se va a luxar a incisión o que la pupila se va a cerrar, colocar retractores de iris (o sistemas de midriasis intraoperatoria similares); hacer la incisión corneal lo más alejada del limbo posible; usar viscoelásticos de alta densidad; inyectar fenilefrina intracamerular si la pupila ya se ha cerrado.

\section{BIBLIOGRAFÍA}

1. KAISER-KuPFER MI, LIPPMAN ME. Tamoxifen retinopathy. Cancer Treat Rep 1978; 62: 315320 .

2. Noureddin BN, Seoud M, Bashshur Z, Salem Z, SAmseddin A, Khalil A. Ocular toxicity in lowdose tamoxifen: a prospective study. Eye 1999; 13: 729-733.

3. Lazzaroni F, Scorolli L, Pizzoleo CF, Savini G, De NigRis A, Giosa F, MEduri RA. Tamoxifen retinopathy: does it really exist? Graefe's Arch Clin Exp Ophthalmol 1998; 236: 669-673.

4. Pavlidis N, Petris C, Briassoulis E, Klouvas G, Psilas C, Rempapis J et al. Clear evidence that long-term low dose tamoxifen treatment can induce ocular toxicity: a prospective study of 63 patients. Cancer 1992; 69: 2961-2964.

5. SeKhar GC, NAGARAJAN R. Ocular toxicity of Tamoxifen. Indian J Ophthalmol 1995; 43: 2326.

6. Alwitry A, Gardner I. Tamoxifen Maculopathy. Arch Ophthalmol 2002; 120: 1402-1402.

7. Tsai DC, Chen SJ, Chiou SH, Lee AF, LeE FL, HSU WM. Should we discontinue tamoxifen in a patient with vision-theatening ocular toxicity related to low-dose tamoxifen therapy? Eye 2003; 17: 276-278.

8. Gorin B, Day R, Costantino JP, Fisher B, REDMOND CK, WiCKERHAM L et al. Long-term tamoxifen citrate use and potential ocular toxicity. Am J Ophthalmol 1998; 125: 493501.

9. Quijada E, Serrano MA, Morales M, García M. Evaluación de efectos secundarios de tamoxifeno a nivel ocular. Arch Soc Canar Oftal 1986-1998; 9: 89-92.

10. Restrepo JF, Guzmán R, Iglesias A. Antimaláricos como drogas inductoras de remisión en la artritis reumatoidea. Revista Colombiana de Reumatología 2002; 9: 62-68.

11. Ferreras A, Pinilla I, Abecia E, Pajarín AB, HonRubia FM. Toxicidad Retiniana Secundaria a Tratamiento con Cloroquina. Archiv Soc Esp Oftalmol 2007; 82: 103-108.

12. Franzco M, Rahman A, McLay J. Early paracentral visual field loss in patients taking hydroxychloroquine. Archiv Ophthalmol 2006; 124: 1729-1733.

13. Eke T, CARR S, Bates AK. Acute galucoma, chronic glaucoma, and serotoninergic drugs. Br J Ophthalmol 1997; 81: 252-253.

14. Kirwan JF, Subak-Sharpe I, Teimory M. Bilateral acute angle closure glaucoma after administration of paroxetine. $\mathrm{Br} \mathrm{J}$ Ophthalmol 1997; 81: 252-254.

15. Reuser T, Flanagan DW, Borland C, BannerJee DK. Acute angle closure galucoma occurring after nebulized bronchodilator treatment with ipratropium bromide and salbutamol. J Roy Soc Med 1992; 85: 499-500.

16. ChANG DF, CAMPELl JR. Intraloperative floppy iris syndrome associated with tamsulosin. J Cataract Refract Surg 2005; 31: 664-673.

17. CHANG DF. Clinical experience with intraoperative floppy-iris syndrome. Results of the 2008 ASCRS member survey. J Cataract Refract Surg 2008; 34: 1201-1209.

18. Cantrell MA, Bream-Rouwenhorst HR, StefFensmeier A, Hemerson P, Rogers M, StAMPER B. Síndrome del iris flácido intraoperatorio 
asociado con los antagonistas de receptores $\beta 1$-Adrenérgicos. Ann Pharmacother 2008; 42 : 558-563.

19. Chadha V, Borooah S, Tey A, Styles C, Singh J. Floppy iris behaviour during cataract surgery: associations and variations. $\mathrm{Br} \mathrm{J}$ Ophthalmol 2007; 91: 40-42.

20. Fontenla JR, VÁzquez XM. SÁnchez J. Fármacos con toxicidad ocular. Jano 1998; 54: 1256-1310. 
